\title{
Asymptotic Analysis of the Shock Wave Ignition of Dust Particles
}

\author{
S. W. BAEK \\ Korea Advanced Institute of Science and Technology, Department of Mechanical Engineering, Aerospace \\ Division, Seoul, Korea
}

and

M. SICHEL and C. W. KAUFFMAN

Department of Aerospace Engineering, The University of Michigan, Ann Arbor, MI 48109

\begin{abstract}
The critical energy required to initiate dust detonations in tubes of finite diameter depends on the structure of the reaction zone, and especially upon the length of the induction zone, which, in turn, is determined by the ignition delay time of the dust particles. To establish the induction zone length in dust detonations, the shock wave ignition of dust particles was investigated theoretically. In the theoretical model the particle acceleration, subsequent convective heating by the hot gas flow, and chemical exothermic surface reaction in the pores as well as on the surface of the particle were considered. An asymptotic analysis for the limit of large activation energy was carried out for spherical particles with constant average values of the gas recovery temperature and the convective heat transfer coefficient. A formula for calculating the ignition delay times was determined and the asymptotic results were compared with a numerical solution of the governing equations and with experimental ignition delay data for coal dust.
\end{abstract}

\section{INTRODUCTION}

The ignition and explosion of clouds of solid dust particles have long been subjects of interest because of their role in accidental explosions in mines and grain elevators. Particularly severe damage occurs when transition to detonation occurs. The energy required for initiation and the propagation characteristics of detonations depend on the structure of the reaction zone and especially upon the induction length, which is determined by the ignition delay time of the fueloxidizer mixture.

In the case of dust detonations the length of the induction zone, which has a direct link to the detonability of the mixture, depends on the actual ignition delay of the individual dust particles behind the leading shock of the detonation front; hence, it is important to measure and develop theoretical models for particle ignition delay behind incident shocks. There have been numerous theoretical and experimental dust particle ignition studies, particularly in the case of coal; however, most of these considered slow ignition with delay times on the order of milliseconds or seconds. Shock-induced ignition delay times are much shorter, typically on the order of about $100 \mu \mathrm{s}$, and the physical processes governing ignition can be quite different from those that are important in slow ignition. Development of a model for the 
shock-induced ignition of dust particles is the subject of the present article.

There have been a number of theoretical studies of the ignition of condensed material based on solid phase, heterogeneous, or gas phase ignition. The solid phase model was used by Bradley [1], who developed the numerical solutions of the equations governing the heating of a reactive, homogeneous solid by a constant flux of energy. Asymptotic analysis for the limit of large activation energy was applied to the ignition of a condensed material by Liñán and Williams [2, 3] and Niioka and Williams [4].

Asymptotic analysis in which the diffusion of the oxidizing gas into the porous solid material is taken into account was also applied by numerous researchers [5-7] to the solution of the heterogeneous ignition problem. The particle ignition analysis of Sichel et al. [13] and Ural [14] was based on a solution of the heat conduction equation for the particle interior, which takes the heat release due to heterogeneous reactions on the surface of internal pores into account. Different ignition sources such as radiation, conductive heating and hot stagnation point flow were considered in these various studies. Gas phase ignition was considered by Kindelan and Williams [8, 9], who applied asymptotic analysis to the radiant ignition of a solid fuel that gasifies endothermically and then reacts exothermically in the gas phase through a one-step Arrhenius reaction.

The Biot number, which is proportional to the ratio of the surface temperature gradient in the solid to that in the gas, plays an important role in the analysis of the shock ignition of particles. In the case of high-conductivity metal particles ignited by a hot gas the Biot number is so small that the particle temperature may be assumed uniform. In particles of low-conductivity material, however, such as coal and oats exposed to the high-velocity gas flow behind an incident shock wave, the Biot number is of order unity because of the intense convective heating. In this case the temperature distribution inside the particle plays a crucial role in ignition, and must be considered in any analysis. In the case of very intense convective heating resulting in very short ignition delay times, the ignition reactions may be confined to a thin high-temperature region near the particle surface.

The evolution of volatiles plays a crucial role in slow ignition and in laminar flame propagation through dusts where ignition times are on the order of milliseconds, and often combustion consists, mainly, of the gas phase oxidation of volatiles. However, Ural [14] has shown that, at least on the basis of slow volatilization rate data, there is insufficient time for significant devolatilization in the ignition of coal, graphite, and char under the conditions generated by an incident shock wave. The effect of volatiles is therefore neglected in the analysis below.

This situation changes when ignition occurs in the stagnant hot gases behind a reflected shock wave, for then the heating rate is relatively slow. In that case it was found from numerical calculations $[13,19]$ that the assumption of a uniform temperature distribution inside the coal particle is acceptable because the rate of heat transfer to the particle interior is then comparable to the surface heating rate from the hot gases. There is then also enough time for the evolution of a substantial fraction of the volatiles.

Ignition delay times of different solid materials behind incident shock waves have been measured by a number of researchers, including Boiko et al. [10], Anderson and Gillespie [11], Hwang and Pillay [12], Sichel et al. [13], Ural [14], and Fox et al. [18]. The gas phase ignition of coal particles in oxygen behind a reflected shock has been observed by Nettleton and Stirling [15].

This article presents a theoretical analysis of the ignition of spherical particles behind an incident shock wave based on the considerations discussed above using high activation energy asymptotics. The results are compared to a numerical solution of the governing equations reported previously [13] and to experimentally measured ignition delay times of coal dust particles. Asymptotic analysis is particularly attractive because it reduces the numerical calculations required, permits rapid evaluation of the influence of model parameters on the results, and should reduce the computation time required when ignition phenomena are incorporated in the simulation of more complex dust cloud phenomena. 


\section{FORMULATION}

Davis et al. [16] observed that the ignition delay time for a mixed sample of different-sized coal particles corresponded to that of the smallest particles, and that under the shock tube test conditions, little interaction occurred among particles of different size. Particle interactions were therefore neglected, and the dust cloud was assumed to consist of monodisperse, spherical particles in the present analysis. Consistent with shock tube ignition delay measurements of small groups of particles such as those reported by Sichel et al. [13] the influence of the particles on the flow behind the incident shock wave was taken to be negligible. However, gas-particle interactions must be taken into account in two phase flows with high particle loading fractions [20].

For a particle in the convective flow behind an incident shock the equation of motion is

$$
\begin{aligned}
\frac{4}{3} & \pi R^{3} \rho_{c} \frac{d V_{p}}{d t} \\
& =\frac{1}{2} C_{d} \rho_{2} \pi R^{2}\left(V_{2}-V_{p}\right)\left|V_{2}-V_{p}\right|,
\end{aligned}
$$

where $\rho_{c}$ and $\rho_{2}$ are the particle and gas densities, $V_{p}$ and $V_{2}$ are the particle and gas flow velocities, $R$ is the particle radius, and $C_{d}$ is the drag coefficient.

When $\left(V_{2}-V_{p}\right)$, the velocity of the gas relative to the particle, is supersonic, a bow shock forms in front of each particle (Fig. 1). As the particle accelerates, the relative velocity decreases below the speed of sound, and the bow shock disappears. Ultimately the relative velocity vanishes altogether.

During acceleration the convective heat trans-

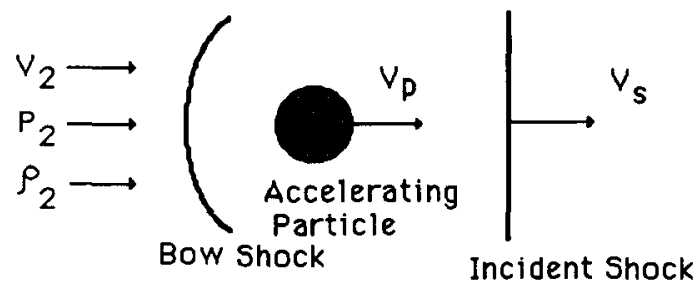

Fig. 1. Schematic diagram of the interaction of a shock wave with a particle. fer from the surrounding gas to the particle causes an increase in the particle temperature. As mentioned above, the Biot number is of the order unity in convected flow behind the shock, so that it is necessary to take the temperature distribution inside the particle into account. To make the analysis tractable the particle temperature distribution and the convective heat flux at the surface are assumed to be spherically symmetric. The unsteady heat conduction equation, including a source term to account for the heat release due to reaction within the particle, is then

$$
\frac{\partial T}{\partial t}=\frac{\alpha}{r^{2}} \frac{\partial}{\partial r}\left(r^{2} \frac{\partial T}{\partial r}\right)+\frac{u^{\prime \prime \prime}(r, t)}{\rho_{c} C},
$$

where $\alpha$ and $C$ are the thermal diffusivity and specific heat of the particle, $T$ is the temperature, and $r$ and $t$ are the radial coordinate and time. The volumetric rate of heat generation, $u^{\prime \prime \prime}$, which accounts for the heat released by the surface reactions within the porous particle, has been taken as

$u^{\prime \prime \prime}=Q \rho_{c} S_{i} P_{\mathrm{O}_{2}} A \exp \left[-\frac{E}{R^{\prime} T}\right]$,

where $S_{i}$ is the internal surface area per unit mass, $Q$ is the heat of combustion, $P_{\mathrm{O}_{2}}$ is the partial pressure of oxygen, $A$ and $E$ are the preexponential factor and activation energy, respectively, and $R^{\prime}$ is the universal gas constant.

Heat transfer at the particle surface can occur through both convection and radiation; however, it can be shown that, for the conditions considered here, the radiative heat flux is negligible compared to the convective flux prior to particle ignition [19]. The boundary condition at the surface of the particle then becomes

$$
k_{\mathrm{c}} \frac{\partial T}{\partial r}(R, t)=h(t)\left[T_{f}(t)-T(R, t)\right],
$$

where $k_{c}$ is the thermal conductivity and $T_{f}$ is the gas recovery temperature at the particle surface. The initial condition and the condition imposed at the particle center because of spherical 
symmetry are given by

$$
\begin{aligned}
& T(r, 0)=T_{i}, \quad r \leq R, \\
& \frac{\partial T}{\partial r}(0, t)=0,
\end{aligned}
$$

where $T_{i}$ is the initial particle temperature.

\section{NUMERICAL CALCULATIONS}

The above system of equations has been solved numerically as described in detail in Refs. 13 and 19. The unsteady heat conduction equation (Eq. 2) is solved by finite differences simultaneously with the trajectory equation (Eq. 1). The heat conduction and trajectory equations are coupled through the film conductance and the recovery temperature $T_{f}$, which, along with the drag coefficient, depends on the current value of the relative velocity $V_{2}-V_{p}$ at each step of the calculation. The surface reactions are initially very slow, while the particle temperature is still low. The maximum temperature occurs at the particle surface and at a fairly well defined time this temperature begins to increase drastically, i.e., temperature runaway occurs. The interval between shock passage over the particle and temperature runaway is taken as the ignition delay time.

Sphere drag and convective heat transfer coefficients are thus required over velocities ranging from supersonic to very low subsonic values in the Stokes flow regime. The empirical correlations given by Henderson [17] were used for the drag coefficient, $C_{d}$. The convective heat transfer coefficient, $h(t)$, used in Eq. 4 depends not only on the Reynolds number but also on the Mach number. An empirical correlation of experimental data developed by Fox et al. [18] was used to determine the convective heat transfer coefficient. In the range $0<\operatorname{Re}<10,000$ and $0<M<6$ this relation is given by

$$
\begin{aligned}
\mathrm{Nu}= & \frac{2 \exp (-M)}{1+17 M / \operatorname{Re}}+0.459 \operatorname{Pr}^{0.33} \operatorname{Re}^{0.55} \\
& \times \frac{1+0.5 \exp (-17 / \operatorname{Re})}{1.5}
\end{aligned}
$$

where $\mathrm{Nu}$ and $\mathrm{Pr}$ are the Nusselt and Prandtl numbers, respectively, and $M$ and $\mathrm{Re}$ are the Mach and Reynolds numbers, respectively, based on the relative velocity and on conditions behind the particle bow shock along the stagnation streamline. The particle diameter was taken as the characteristic length. The initial temperature of the particle was taken as $T=T_{i}=295 \mathrm{~K}$ in the calculations presented here.

\section{ASYMPTOTIC ANALYSIS}

Liñán and Williams [2] investigated the ignition of a semiinfinite reactive solid by a constant energy flux at the surface using high activation energy asymptotics. Here this asymptotic method will be applied to the determination of the ignition delay time of a solid spherical particle exposed to a hot convective flow as described by the formulation presented above. A key assumption, which is discussed in more detail later, is that the recovery temperature and heat transfer coefficient are constant at suitably determined average values over the ignition delay period.

It is now appropriate to introduce dimensionless variables. Thus, using

$\theta=\frac{r T}{R T_{i}}, \quad \tau=\frac{\alpha t}{R^{2}}, \quad \xi=\frac{r}{R}$,

the heat conduction equation (Eq. 2) becomes

$$
\frac{\partial \theta}{\partial \tau}=\frac{\partial^{2} \theta}{\partial \xi^{2}}+A^{\prime} \xi \exp \left(-\frac{E^{\prime} \xi}{\theta}\right),
$$

where

$$
E^{\prime}=\frac{E}{R^{\prime} T_{i}}, \quad A^{\prime}=\frac{Q \rho_{c} S_{i} P_{\mathrm{O}_{2}} A R^{2}}{k_{c} T_{i}}
$$

In terms of the new variables the initial condition (Eq. 5a) becomes

$$
\theta(\xi, 0)=\frac{r}{R}=\xi
$$

The numerical solution of Eqs. 1-5 discussed above and the ignition delay studies of Lee et al. [20] in which the temporal variation of the con- 
vective heat transfer coefficient, $h$, and gas recovery temperature, $T_{f}$, were taken into account, showed that the temperature at the center of the particle remained, essentially, at the initial value $T_{i}$ during the ignition delay period. This effect is graphically illustrated by the temperature distribution histories shown in Ref. 20. Consequently, it is reasonable to simplify the asymptotic problem by replacing the symmetry condition (Eq. 5b) with $T(0, t)=T_{i}$. The symmetry condition at the center and the particle surface boundary condition then become

$\theta(0, \tau)=0$,

$$
\frac{\partial \theta}{\partial \xi}(1, \tau)-\theta(1, \tau)=B_{i}\left[\frac{T_{f}}{T_{i}}-\theta(1, \tau)\right],
$$

where $B_{i}=h R / k_{c}$.

In dealing with the Arrhenius term in the energy equation it is convenient to introduce the parameter $\theta_{1}=E^{\prime} / \ln A^{\prime}$ so that the dimensionless heat conduction equation (Eq. 7) becomes

$$
\frac{\partial \theta}{\partial \tau}=\frac{\partial^{2} \theta}{\partial \xi^{2}}+\exp \left[\frac{E^{\prime}}{\theta_{1}}\left(\frac{\theta-\theta_{1} \xi}{\theta}\right)\right] .
$$

The condition $\theta_{1}>1$ must be satisfied if ignition is to occur at a well-defined ignition temperature $\theta_{c}$. This requirement follows from the fact that in the asymptotic limit $E^{\prime} / \theta_{1} \Rightarrow \infty$, the reaction term will be exponentially small for $\theta<\theta_{1}$ and exponentially large for $\theta>\theta_{1}$ at the surface of the particle, i.e., at $\xi=1$. When $\theta(1, \tau)-\theta_{1}$ becomes of the order of $\theta_{1} / E^{\prime}$, the reaction term in Eq. 12 becomes important and it is anticipated that transition to ignition will occur.

To lowest order in the small parameter $\theta_{1} / E^{\prime}$, for values of $\tau$ so small that $\theta<\theta_{1}$ holds everywhere in the solid, the exponential term in Eq. 12 will be negligibly small, so that this equation reduces to the nonreactive form:

$$
\frac{\partial \theta}{\partial \tau}=\frac{\partial^{2} \theta}{\partial \xi^{2}}
$$

with the same initial and boundary conditions, (Eqs. 9-11). The solution corresponds to the initial stage of inert heating when no chemical reaction is involved and is found to be $\theta=\theta_{I}$, with

$$
\theta_{I}=\frac{B \xi}{1+Z}+\sum_{n=1}^{\infty} \beta_{n} \sin \left(\lambda_{n} \xi\right) \exp \left(-\lambda_{n}^{2} \tau\right)
$$

where

$B=\frac{B_{i} T_{f}}{T_{i}}, \quad B_{i}=\frac{h R}{k_{c}}, \quad Z=B_{i}-1$.

The eigenvalue $\lambda_{n}$ is determined by the relation

$\tan \lambda_{n}=-\frac{\lambda_{n}}{Z}$

and the Fourier coefficient $\beta_{n}$ is given by

$\beta_{n}=\frac{4\left(1-\frac{B}{1+Z}\right)\left(\frac{\sin \lambda_{n}}{\lambda_{n}}-\cos \lambda_{n}\right)}{2 \lambda_{n}-\sin \left(2 \lambda_{n}\right)}$.

From the discussion above it follows that to lowest order in $\theta_{1} / E^{\prime}$ ignition will occur when at some time $\tau_{c}$ the inert temperature $\theta_{I}$ at the surface $\xi=1$ reaches the value $\theta_{1}$ because thermal runaway then occurs at the surface. To this order the ignition temperature $\theta_{c}$ and the ignition time $\tau_{c}$ are determined by the relation

$$
\begin{aligned}
\theta_{c}=\theta_{1}=\theta_{1}\left(1, \tau_{c}\right)= & \frac{B}{1+Z} \\
& +\sum_{n=1}^{\infty} \beta_{n} \sin \left(\lambda_{n}\right) \\
& \cdot \exp \left(-\lambda_{n}^{2} \tau_{c}\right) .
\end{aligned}
$$

This lowest order result ignores the fact that the transition from nonreactive to reactive heating does not occur discontinuously at the surface. To analyze the transition from inert heating to incipient reaction, the deviation of the nondimensional temperature from its inert value, $\theta_{I}$, is introduced and is expressed as

$\Phi=\theta-\theta_{I}$.

The governing equation for $\Phi$ can then be found 
by substituting Eq. 16 into Eq. 12:

$$
\begin{aligned}
\frac{\partial \Phi}{\partial \tau}= & \frac{\partial^{2} \Phi}{\partial \xi^{2}}+\xi A^{\prime} \\
& \times \exp \left(\frac{E^{\prime}}{\theta_{c}}\right) \exp \left[\left(\frac{E^{\prime}}{\theta_{c}}\right)\left(\frac{\theta_{I}-\theta_{c} \xi+\Phi}{\theta_{I}+\Phi}\right)\right]
\end{aligned}
$$

and the initial and boundary conditions now take the forms

$$
\begin{aligned}
\frac{\partial \Phi}{\partial \xi}(1, \tau) & =\left(1-B_{i}\right) \Phi(1, \tau), \\
\Phi(0, \tau) & =0, \\
\Phi(\xi, 0) & =0 .
\end{aligned}
$$

The maximum temperature always occurs at the surface of the solid as shown by Baek [19]. Thus, expanding the inert solution (Eq. 14) about $\tau=\tau_{c}, \xi=1$, using Eq. 15, gives

$\theta_{I}=\theta_{c}+\sum_{n=1}^{\infty}\left[-C_{n}(1-\xi)+D_{n}\left(\tau-\tau_{c}\right)\right]$,

where

$C_{n}=\beta_{n} \lambda_{n} \cos \left(\lambda_{n}\right) \exp \left(-\lambda_{n}^{2} \tau_{c}\right)$,

$D_{n}=-\beta_{n} \lambda_{n}^{2} \sin \left(\lambda_{n}\right) \exp \left(-\lambda_{n}^{2} \tau_{c}\right)$.

Substituting Eq. 19 into Eq. 17 and retaining only the first nonvanishing terms in $1-\xi$ the equation for $\Phi$ becomes

$$
\begin{aligned}
\frac{\partial \Phi}{\partial \tau}=\frac{\partial^{2} \Phi}{\partial \xi^{2}}+A^{\prime} & \\
& \times \exp \left(-\frac{E^{\prime}}{\theta_{c}}\right) \exp \left[( \frac { E ^ { \prime } } { \theta _ { c } ^ { 2 } } ) \left((\xi-1) \sum_{n=1}^{\infty} C_{n}\right.\right. \\
& \left.\left.\quad+\left(\tau-\tau_{c}\right) \sum_{n=1}^{\infty} D_{n}+\Phi\right)\right]
\end{aligned}
$$

To obtain an asymptotic expansion of the solution to the equation for $\Phi$ as $E^{\prime} \Rightarrow \infty$ two separate regions are considered. If the variables (1 $\xi)$ and $\left(\tau-\tau_{c}\right)$ are stretched so that the terms containing them in the second exponential in Eq. 21 are of the same order it is found that the unsteady term $\partial \Phi / \partial \tau$ is of higher order than the diffusion term $\partial^{2} \Phi / \partial \xi^{2}$. Accordingly, there is an inner reactive-diffusive region near the particle surface where an appropriate expansion for $\Phi$ has the form

$$
\begin{aligned}
\Phi= & \delta^{2} \Psi_{0}(\chi, \sigma)+\delta^{3} \Psi_{1}(\chi, \sigma) \\
& +\delta^{4} \Psi_{2}(\chi, \sigma) \cdots,
\end{aligned}
$$

with the expansion parameter

$\delta=\theta_{c} E^{\prime-1 / 2}$.

The stretched variables are then given by

$\sigma=\delta^{-2} K_{2}\left(\tau-\tau_{c}\right)+b_{0}$,

$\chi=\delta^{-2} K_{1}(1-\xi)$,

with

$$
K_{1}=\sum_{n=1}^{\infty} C_{n} \quad K_{2}=\sum_{n=1}^{\infty} D_{n} .
$$

$b_{0}$ is defined by the expansion

$$
\begin{aligned}
b & =\ln \left[A^{\prime} K_{2}^{-1 / 2} K_{1}^{-1} \delta \exp \left(-\frac{E^{\prime}}{\theta_{c}}\right)\right] \\
& =b_{0}+b_{1} \delta+b_{2} \delta^{2}+\cdots .
\end{aligned}
$$

The second region is an outer nonreactive transient-diffusive region in the interior of the particle where the reactive term is negligible. Here the appropriate expansion for $\Phi$ takes the form:

$$
\begin{aligned}
\Phi= & \delta^{2} \omega_{0}(\eta, \sigma)+\delta^{3} \omega_{1}(\eta, \sigma) \\
& +\delta^{4}(\eta, \sigma)+\cdots,
\end{aligned}
$$

where

$\eta=\delta K_{2}^{1 / 2} K_{1}^{-1} \chi=\delta^{-1} K_{2}^{1 / 2}(1-\xi)$.

In terms of the stretched variable $\eta$ the two derivatives $\partial \Phi / \partial \tau$ and $\partial^{2} \Phi / \partial \xi^{2}$ become of the same order in the transient-diffusive region.

Substituting Eqs. 22 and 23 in Eq. 21, the governing equation in the inner reactive region 
becomes

$$
\begin{aligned}
& \frac{\partial^{2} \Psi_{0}}{\partial \chi^{2}}+\delta \frac{\partial^{2} \Psi_{1}}{\partial \chi^{2}}+\delta^{2} \frac{\partial^{2} \Psi_{2}}{\partial \chi^{2}} \\
& -\delta^{2} K_{1}^{-2} K_{2} \frac{\partial \Psi_{0}}{\partial \sigma}+O\left(\delta^{3}\right) \\
& =-\delta K_{1}^{-1} K_{2}^{1 / 2} \exp \left[\sigma-\chi+\Psi_{0}\right. \\
& \left.+\delta \Psi_{1}+\delta b_{1}+O\left(\delta^{2}\right)\right],
\end{aligned}
$$

and it can be seen that the unsteady term is of higher order than the reactive and diffusive terms.

From Eq. 18 it follows that in terms of the stretched variables the initial condition becomes

$\Psi_{j}(\chi, \sigma) \Rightarrow 0$ as $\sigma \Rightarrow-\infty$,

$$
j=0,1,2, \ldots,
$$

while the boundary conditions become

$$
\begin{aligned}
& \frac{\partial \Psi_{0}}{\partial \chi}(0, \sigma)=\frac{\partial \Psi_{1}}{\partial \chi}(0, \sigma)=0 \\
& \Psi_{0}(\chi, \sigma), \Psi_{1}(\chi, \sigma) \Rightarrow 0 \text { as } \chi \Rightarrow \infty
\end{aligned}
$$

To lowest order in the inner region it now follows from Eq. 25 that $\Psi_{0}$ satisfies the equation

$\frac{\partial^{2} \Psi_{0}}{\partial \chi^{2}}=0, \quad$ with $\frac{\partial \Psi_{0}}{\partial \chi}(0, \sigma)=0$.

The corresponding solution is $\Psi_{0}=\Psi_{0}(\sigma)$, where $\Psi_{0}(\sigma)$ is a function of $\sigma$ to be determined from the matching conditions. To next order, Eq. 25 yields the following equation for $\Psi_{1}$ :

$\frac{\partial^{2} \Psi_{1}}{\partial \chi^{2}}=-K_{1}^{-1} K_{2}^{1 / 2} \exp \left[\sigma-\chi+\Psi_{0}(\sigma)\right]$,

with the boundary condition

$$
\frac{\partial \Psi_{1}}{\partial \chi}(0, \sigma)=0
$$

The solution of this equation is

$$
\begin{aligned}
\Psi_{1}= & -K_{1}^{-1} K_{2}^{1 / 2}\left[\exp (-\chi)+\chi+f_{1}(\sigma)\right] \\
& \times\left[\exp \left(\sigma+\Psi_{0}\right)(\sigma)\right]
\end{aligned}
$$

where the function $f_{1}(\sigma)$ is to be determined from the matching conditions between the inner and outer solutions.

Substituting Eq. 24 and the stretched variable $\eta$ into Eq. 21, the equation for the outer region becomes

$$
\begin{aligned}
\frac{\partial \omega_{0}}{\partial \sigma} & -\frac{\partial^{2} \omega_{0}}{\partial \eta^{2}}+\delta\left(\frac{\partial \omega_{1}}{\partial \sigma}-\frac{\partial^{2} \omega_{1}}{\partial \eta^{2}}\right)+O\left(\delta^{2}\right) \\
= & \delta^{-1} K_{1} K_{2}^{-1 / 2} \\
& \times \exp \left(-\delta^{-1} K_{1} K_{2}^{-1 / 2} \eta+O(1)\right) .
\end{aligned}
$$

From Eq. 18 it follows that the coefficients of the outer expansion satisfy the initial conditions

$\omega_{j}(\eta, \sigma) \rightarrow 0$ as $\sigma \rightarrow-\infty, \quad j=0,1,2, \cdots$, and the boundary conditions are

$\omega_{j}\left(\delta^{-1} K_{2}^{-1 / 2}, \sigma\right)=0, \quad j=0,1,2, \cdots$.

The matching conditions between the inner and outer regions must now be found. The one term outer expansion, i.e., $\omega_{0}(\eta, \sigma)$ is first expanded in terms of the inner variable $\chi$, so that

$$
\begin{aligned}
\omega_{0}(\eta, \sigma)= & \omega_{0}(0, \sigma)+\delta K_{2}^{1 / 2} K_{1}^{-1} \chi \\
& \times \frac{\partial \omega_{0}}{\partial \eta}(0, \sigma)+\cdots \\
= & \omega_{0}(0, \sigma)+\eta \frac{\partial \omega_{0}}{\partial \eta}(0, \sigma) .
\end{aligned}
$$

The two-term inner expansion, i.e., $\Psi_{0}(\chi, \sigma)$ $+\delta \Psi_{1}(\chi, \sigma)$, expressed in terms of outer variables becomes

$$
\begin{aligned}
& \Psi_{0}(\sigma)+\delta\left\{-K_{1}^{-1} K_{2}^{1 / 2}\left[\exp \left(\frac{K_{2}^{-1 / 2} K_{1} \eta}{\delta}\right)\right.\right. \\
& \left.\left.+\frac{K_{2}^{-1 / 2} K_{1} \eta}{\delta}+f_{1}(\sigma)\right] \exp \left[\sigma+\Psi_{0}(\sigma)\right]\right\}
\end{aligned}
$$


From this result it follows that the one term outer expansion of the inner expansion is

$$
\Phi=\Psi_{0}(\sigma)-\eta \exp \left[\sigma+\Psi_{0}(\sigma)\right]+\cdots .
$$

Matching now requires that Eqs. 27 and 28 be equal, which then leads to the relations

$$
\begin{aligned}
& \omega_{0}(0, \sigma)=\Psi_{0}(\sigma), \\
& \frac{\partial \omega_{0}}{\partial \sigma}(0, \sigma)=-\exp \left[\sigma+\omega_{0}(0, \sigma)\right] .
\end{aligned}
$$

Keeping only the lowest-order terms, Eq. 26 reduces to the following partial differential equation for the outer expansion coefficient $\omega_{0}(\eta, \sigma)$ :

$\frac{\partial \omega_{0}}{\partial \sigma}-\frac{\partial^{2} \omega_{0}}{\partial \eta^{2}}=0$.

Not surprisingly, the lowest-order outer expansion coefficient satisfies the nonreactive diffusion equation with the boundary condition (Eq. 29) determined from matching with the inner reactive solution. As already shown, it follows from Eq. 18 that in the limit $\delta \Rightarrow 0, \omega_{0}$ satisfies the initial condition

$\omega_{0}(\eta, \sigma) \rightarrow 0$ as $\sigma \rightarrow-\infty$,

while the additional boundary condition becomes

$\omega_{0}(\eta, \sigma) \rightarrow 0$ as $\eta \rightarrow \infty$.

Equations 29-32 were solved by Liñán and Williams [2] in their study of the ignition of a semiinfinite solid combustible material, even though the problem that they considered is different from that considered here. Thus, although they considered a semiinfinite solid material exposed to a constant heat flux, the present study is concerned with the convective heating of a spherical particle. Their solution showed that thermal runaway, i.e., $\omega_{0} \Rightarrow \infty$, occurred at the finite time $\sigma=b_{0}=-0.431$. Using the result $\sigma=$ -0.431 , the ignition time $\tau_{c}$ can be calculated from Eqs. 15 and 23.

The influence of particle acceleration enters the asymptotic analysis implicitly through the recovery temperature $T_{f}$ and film conductance $h$, which must be specified in Eq. 11 for the particle sur- face boundary condition. These parameters are treated as constants in the asymptotic analysis; however, $T_{f}$ as well as $h$ change as the particles are accelerated to the gas velocity behind the incident shock wave, and this variation was taken into account in the numerical analysis as described above. In the asymptotic analysis suitable average values were used for $h$ and $T_{f}$. The particle trajectory equation (Eq. 1) can be integrated independently of the heat conduction equation so that the variation of $T_{f}$ and $h$ with time during particle acceleration are readily determined. The average values of $T_{f}$ and $h$ were then evaluated using

$\bar{T}_{f}=\frac{1}{t_{i}} \int_{0}^{t_{i}} T_{f} d t, \quad \bar{h}=\frac{1}{t_{i}} \int_{0}^{t_{i}} h d t$.

The ignition delay time $t_{i}$ is determined using the asymptotic analysis, which in turn depends on these average values so that iteration was required to obtain the solution.

\section{RESULTS AND DISCUSSION}

Ignition delay times of 53- $\mu \mathrm{m}$-diameter coal particles computed using the asymptotic analysis described above are compared to experimentally measured and numerically computed delay times in Fig. 2. The experimental measurements, which were conducted using air as the oxidizing gas, are described in detail in Refs. 13 and 19. The dust particles were injected into the shock tube just before arrival of the shock wave using either an air injector or a special inertial injector, and the data obtained using both methods of injection are shown. The ignition delay time was determined by measuring the particle radiation using a photomultiplier tube.

The following physical and kinetic data for the coal dust were used in the calculations and were taken from Badzioch et al. [21], Gan et al. [22], and Field et al. [23]:

Density, $\rho_{c}=1.2 \mathrm{~g} / \mathrm{cm}^{3}$

Thermal conductivity, $\lambda=0.00212 \mathrm{cal} / \mathrm{cm} \mathrm{K} \mathrm{s}$

Spec. heat, $C=0.236 \mathrm{cal} / \mathrm{gm} \mathrm{K}$

Heat of combustion, $Q=8559 \mathrm{cal} / \mathrm{g}$ 


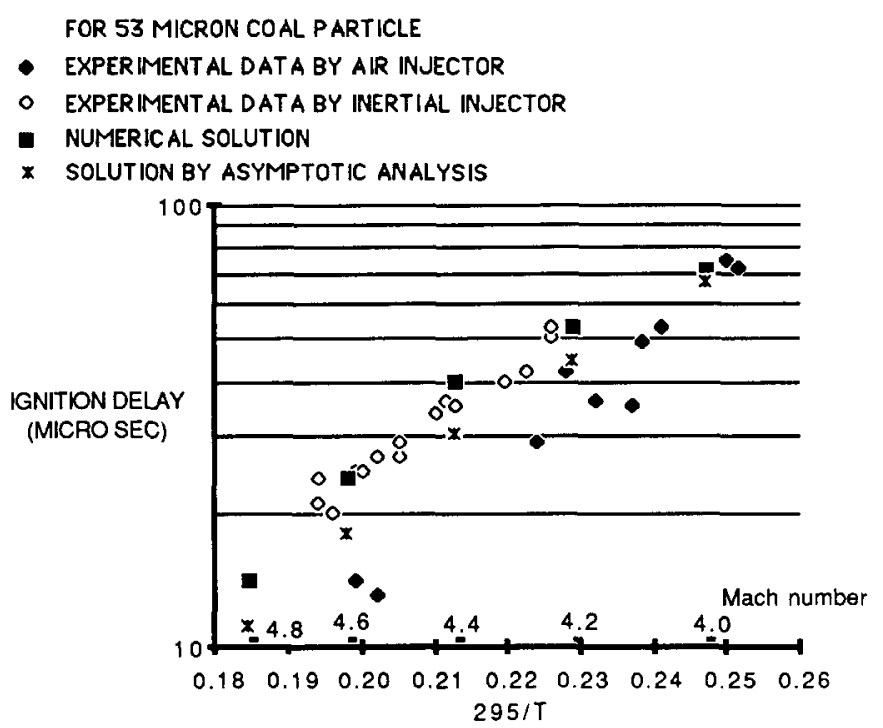

Fig. 2. Comparison of asymptotically computed and measured ignition delays for coal dust.

Int. surf. area, $S_{i}=4.26 \times 10^{6} \mathrm{~cm}^{2} / \mathrm{g}$

Activation energy, $E=35.7 \mathrm{kcal} / \mathrm{gmol}$

Preexponential factor, $A=8.71 \times 10^{3} \mathrm{~g} / \mathrm{cm}^{2} \mathrm{~s}$ atm

Ignition delay times are plotted on a logarithmic scale versus the inverse of the postshock gas static temperature nondimensionalized by the initial temperature of $295 \mathrm{~K}$. The corresponding incident shock wave Mach numbers are also indicated on the abscissa. This form of representation is used to plot Arrhenius rate law data since the resultant plots are then linear. The present results also lie on an almost straight line even though dust ignition involves a complex combination of physical and chemical processes. This behavior also has been observed in the case of droplet ignition, suggesting that these complex processes can, in some cases, be represented by an equivalent Arrhenius rate law.

The theoretically computed ignition delay times using the high-activation-energy asymptotic analysis were in good agreement with the numerical solution of the governing equations and with the experimentally measured values. The calculations were performed using physical constants from independent sources in the literature. In view of the many approximations used in the analysis and the difficulties involved in measuring ignition de- lay times [13] these results are encouraging and suggest that ignition delay times of dust particles behind incident shock waves can reasonably be predicted using either the asymptotic or numerical analysis described above.

From the analysis it can be seen that for the conditions considered here the heat-up time of the particles is the main factor governing the incident shock wave ignition of solid particles. The asymptotic analysis leads to the result that in terms of the transformed variables particle ignition is governed by the same system of one dimensional heat conduction equations solved by Linan and Williams [2] in their study of the ignition of a semiinfinite solid. This reflects the fact that behind an incident shock, prior to ignition, particle heating is restricted to a thin layer near the particle surface so that the particle, locally, behaves like a semiinfinite solid.

The use of constant average values for the film coefficient and recovery temperature greatly complicates the asymptotic analysis but is necessary because there is no simple analytical way to represent the particle trajectories during acceleration to the fluid velocity behind the incident shock. Even so, a major advantage of the asymptotic analysis is that it eliminates the need to numerically integrate the heat conduction equation for the particle interior in order to compute ignition 
delay times, and the validation of this approach is an important contribution of the present article. Use of the asymptotic solution should significantly reduce computing time in the numerical simulation of the shock ignition of more complex dust cloud configurations. In cases in which a characteristic particle acceleration time is much less than a characteristic chemical time it may be quite reasonable to equate the constant surface conditions to those at the end of particle acceleration, while use of conditions immediately behind the shock may be appropriate if the particle acceleration time is very long.

The main result of this article is to show that high activation energy asymptotic analysis can be used to determine the ignition delay times of combustible dust particles behind incident shock waves.

This work was partially sponsored by the National Science Foundation under Grant CPE-8023865, R. Rostenbach, project monitor and by The Air Force Office of Scientific Research under AFOSR Grant No. 79-0093, B. J. Wolfson, project monitor.

\section{REFERENCES}

1. Bradley, H. H., Jr., Combust. Sci. Technol. 2:11 (1970).

2. Liñán, A., and Williams, F. A., Combust. Sci. Technol. 3:91 (1971).

3. Liñán, A., and Williams, F. A., Combust. Flame 18:85 (1972).

4. Niioka, T., and Williams, F. A., Combust. Flame 29:43 (1977).

5. Kindelan, M., and Liñán, A., AFOSR Technical Report
73-2535, European Office of Aerospace Research and Development, 1976.

6. Bradley, H. H., Jr., and Williams, F. A., Combust. Sci. Technol. 2:41 (1970).

7. Niioka, T., Combust. Sci. Technol. 18:207 (1978).

8. Kindelan, M., and Williams, F. A., Acta Astronaut. 2:955 (1976).

9. Kindelan, M., and Williams, F. A., Combust. Sci. Technol. 16:47 (1977).

10. Boiko, V. M., Fedorov, A. V., Fomin, V. M., Papyrin, A. N., and Soloukhin, R. I., in Prog. Astronaut. Aeronaut. 87:71 (1981).

11. Anderson, W. H., and Gillespie, F. L., Combust. Sci. Technol. 24:35 (1980).

12. Hwang, C. C., and Pillay, S., Combust. Sci. Technol. 17:241 (1978).

13. Sichel, M., Baek, S. W., Kauffman, C. W., Maker, B., Nicholls, J. A., AIAA J. 23:1374 (1985).

14. Ural, E. A., Ph.D. thesis, The University of Michigan, Ann Arbor, MI, 1981.

15. Nettleton, M. A., and Stirling, R., Proc. Roy. Soc. Lond. A 300:62 (1967).

16. Davis, W. P., Baer, A. D., and Ryan, N. W., Combust. Flame 58:201 (1984).

17. Henderson, C. B., AIAA J. 14:707 (1976).

18. Fox, T. W., Rackett, C. W., and Nicholls, J. A., Proceedings of the 11th International Symposium on Shock Tubes and Waves, University of Washington, 1977.

19. Baek, S. W., Ph.D thesis, The University of Michigan, Ann Abor, MI, 1985.

20. Lee, F. P., Sichel, M., Kauffman, C. W., and Nicholls, J. A., AIAA J. 24:1811 (1986).

21. Badzioch, S., Gregory, D. R., and Field, M. A., Fuel 43:267 (1964).

22. Gan, H., Nandi, S. P., and Walker, P. L., Fuel 51:272 (1972).

23. Field, M. A., Gill, D. W., Morgan, P. P., and Hawskley, P. G., Combustion of Pulverized Coal, The British Coal Utilization Research Association, Leatherhead, 1967.

Received 28 October 1988; revised 25 September 1989 